\title{
Espacios de participación estudiantil y perspectiva de derechos en escuelas secundarias de una ciudad intermedia de la provincia de Córdoba
}

\author{
MARÍA SILVINA BAIGORRIA(1) \\ CLAUDIO CÉSAR ACOSTA ${ }^{(2)}$ \\ MARÍA FLORENCIA CASTILLO(3) \\ GIULIANA LARA MARCLÉ(4)
}

Fecha de recepción: 28/12/2019

Fecha de aceptación: 03/07/2020

Resumen. Este trabajo presenta resultados parciales de un proyecto de investigación que tuvo como propósito cardinal estudiar la construcción de la perspectiva de derechos en tres escuelas secundarias insertas en contextos de desigualdad social en una ciudad intermedia de la provincia de Córdoba. Se identificaron los sentidos que los y las jóvenes construyen en relación con las prácticas que las escuelas institucionalizan como potenciadoras de derechos, en el proceso dinámico y complejo de construcción de ciudadanía en conexión con un contexto de reemergencia neoconservadora que disputa sentidos sobre la perspectiva de derechos en las escuelas. Se analizan las prácticas de los y las jóvenes en los centros de estudiantes de las tres escuelas y el espacio de las asambleas escolares para luego problematizar cómo estas tensionan y /o potencian la perspectiva de derechos que las instituciones asumen en sus proyectos institucionales.

Palabras clave. derechos · jóvenes . ciudadanía $\cdot$ escuelas
(1) Universidad Nacional de Río Cuarto (UNRC). Especialista en Docencia Universitaria y Licenciada en Psicopedagogía. Docente de la cátedra de Sociología de la Educación de la Facultad de Ciencias Humanas de la UNRC. Directora del Proyecto de investigación: La trama de los derechos en escuelas de nivel medio: ¿Conquistas, concesiones o mandatos? Tensiones entre la contrahegemonía y la reproducción. Un estudio en tres escuelas de nivel medio de la ciudad de Río Cuarto (2016-2019) Aprobado por SECYT. UNRC. Directora de becas de ayudantía de investigación de SECYT-UNRC

sbaigorria@hum.unrc.edu.ar

(2) Universidad Nacional de Río Cuarto (UNRC). Maestrando de la Maestría en Ciencias Sociales de la UNRC. Profesor de Historia. Docente de las cátedras de Sociología, Sociología de la Educación y Política Educativa de la Facultad de Cs. Humanas de la UNRC. Co- director de becas de ayudantías de investigación de SECYTUNRC.

cacosta@hum.unrc.edu.ar

(3) Universidad Nacional de Río Cuarto (UNRC). Estudiante de la Licenciatura en Psicopedagogía. Ayudante de la cátedra de Sociología de la educación. Becaria de investigación Prácticas de participación juvenil y perspectiva de derechos. «Un estudio en centros de estudiantes de tres escuelas secundarias de la ciudad de Río Cuarto en contextos desiguales». 2018-2019. chinaacastilloo@gmail.com

(4) Universidad Nacional de Río Cuarto (UNRC). Estudiante de la Licenciatura en Psicopedagogía. Ayudante de la cátedra de Sociología de la educación. Becaria de investigación «La perspectiva de derechos en el espacio escolar: construcciones juveniles. Estudio en una escuela secundaria de la ciudad de Rio Cuarto». 20I8-20I9. giulianalaramarcle@gmail.com 


\section{Areas for Student Participation and the Rights Perspective in Secondary Schools}

\begin{abstract}
This paper presents partial results of a research project aimed at studying the construction of the rights perspective in three secondary schools dealing with social inequality contexts in an intermediate city of the province of Córdoba. The present work addresses the senses conveyed by youngsters in relation to the practices institutionalized by schools as rights enhancers in the dynamic and complex process of constructing citizenship within a context of neoconservative
\end{abstract}

re-emergence that dispute over senses about the rights perspective in schools. The practices of youngsters in student councils of three schools and the areas for school assemblies are analyzed so as to question how they strengthen and/or put a strain on the rights perspective taken on for institutional projects.

Keywords. Rights $\cdot$ youngsters $\cdot$ citizenship $\cdot$ schools
(5) Becas de Investigación "Centenario de la Reforma " (BECER 20I8) SECYT. UNRC. «La perspectiva de derechos en el espacio escolar: construcciones juveniles.

Estudio en una escuela secundaria de la ciudad de Rio Cuarto» y «Prácticas de participación juvenil y perspectiva de derechos. Un estudio en centros de estudiantes de tres escuelas secundarias de la ciudad de Río Cuarto en contextos desiguales». Ambos trabajos de becas se inscriben en el Proyecto de Investigación PPI «La trama de los derechos en escuelas de nivel medio: ¿Conquistas, concesiones o mandatos? Tensiones entre la contra-hegemonía y la reproducción. Un estudio en tres escuelas de nivel medio de la ciudad de Río Cuarto" (2016-20I8) Aprobado por SECYT. UNRC

\section{Introducción}

Este trabajo presenta resultados parciales de un proyecto de investigación que tuvo como propósito estudiar la construcción de la perspectiva de derechos en tres escuelas secundarias insertas en contextos de desigualdad social en una ciudad intermedia de la provincia de Córdoba. Se presentan aquí algunas reflexiones a partir del trabajo desarrollado de dos becas de ayudantías de investigación ${ }^{(5)}$ que analizaron las prácticas de participación estudiantil en tres escuelas secundarias durante el año 2018. Se identificaron los sentidos que los y las jóvenes construyen en relación con las prácticas que las escuelas institucionalizan como potenciadoras de derechos en el proceso dinámico y complejo de construcción de ciudadanía. En el marco de estos trabajos se estudió particularmente dos espacios de participación juvenil en las escuelas: los centros de estudiantes y las asambleas escolares (espacio existente en una de las escuelas).

El artículo aborda algunas de las notas características de las luchas de sentido sobre la perspectiva de derechos en el actual contexto de reemergencia neoconservadora, para luego problematizar cómo los y las jóvenes significan los espacios de participación escolar. Nos orientaron interrogantes tales como: ¿Qué procesos de construcción de derechos se configuran en escuelas insertas en contextos desiguales? ¿Cuáles son las prácticas que 
los y las jóvenes estudiantes realizan en relación a esta perspectiva? ¿Qué sentidos construyen sobre las prácticas institucionalizadas como ampliadoras o potenciadoras de derechos? Pretendemos así aportar algunas reflexiones desde el campo de la Sociología de la Educación crítica en relación a esta temática.

\section{Derechos y ciudadanía en la escuela}

En estos últimos años la embestida de gobiernos neoconservadores en América del Sur (Chile, Brasil, Ecuador y en Argentina con la administración de Cambiemos, entre otras experiencias similares) ha potenciado un proceso de desmantelamiento de los avances en torno a los derechos de niñas, niños y jóvenes; en este marco, las políticas arremeten contra la «agenda de derechos" y promueven un retroceso de las conquistas logradas (Rodríguez 2018). Cabe destacar que, a pesar de estas reemergencias neoconservadoras, las luchas sociales locales y globales, en clave de procesos históricos, habían logrado sancionar un conjunto de ordenamientos jurídicos que regularon y respaldaron la perspectiva de derechos, más o menos distantes de las prácticas territoriales e institucionales que reaseguraran su cumplimiento efectivo. A pesar de la adhesión al nuevo paradigma de derechos, las instituciones estaban aún lejos de generar los cambios sustantivos que posibilitaran reducir, por ejemplo, las desigualdades acrecentadas por la implementación de políticas neoliberales de los '90 (Llobet 2013). Si bien no se desconoce el avance fundamental que significó este conjunto de normas y tratados, estos han tenido un eco diferente en territorios heterogéneos y desiguales y en las diferentes experiencias escolares, a veces para generar procesos inclusivos, otras para contrarrestarlos.

Según Barna (2013) la perspectiva de los derechos al convertirse «herramienta potente, versátil y maleable» encuentra terreno fértil para ser utilizada estratégicamente por grupos y sectores con antagónicas concepciones y posicionamientos sobre niñeces y adolescencias políticamente opuestas. Es un campo de disputa cuyas expresiones se dan cita de manera distinta según las dinámicas sociales y las diversas coyunturas políticas, se pretende 
fundamentar posicionamientos políticos y epistemológicos frente a niñas, niños y jóvenes en donde se ponen en juego valores y cosmovisiones a veces opuestas. Se genera entonces una paradoja: bajo el paraguas de los derechos se cobija un abanico de posiciones contrarias. Entonces, la sola referencia a la perspectiva de derechos no implica asumir una posición intrínsecamente emancipadora, pues es foco de luchas de sentido y de apropiación para su definición.

Por otra parte, G. Magistris (2013) advierte sobre la emergencia de un «magnetismo incuestionable, rígido, cerrado en sí mismo» acerca de los derechos, expresado en un discurso casi naturalizado que ha ganado legitimidad en el espacio escolar, con carácter prescriptivo de prácticas. Su carácter abstracto y totalizante dificulta considerar las condiciones de clase social, de género y de etnia para comprender las desiguales formas de cumplimiento y vulneración de los derechos de los y las jóvenes. Este carácter totalizante promueve también la naturalización de la perspectiva de derechos en las escuelas y obtura la visibilización de cómo se producen prácticas y sentidos en contextos particulares.

En relación a la comprensión de ciudadanía juvenil plena Morales y Magistris (2018) nos insinúan sobre el carácter limitado y restringido que resultó el derecho a la participación manifiesto en la Convención de los Derechos del Niño. Se habilitó así una versión instrumental de la misma, dando lugar a una ciudadanía de segundo nivel o una existencia pre-ciudadana: acentuado por un enfoque de carácter proteccionista que debilita y restringe avances a una integralidad que amplíe las posibilidades de participación. (Baratta en Magistris 2018). Para Fuentes y Nuñez (2015) la ciudadanía es un significante amplio, asociado a la participación, la democracia, la convivencia, la disciplina, la autoridad, la politicidad. En Argentina, en las últimas décadas la ciudadanía en la escuela adquirió nuevos sentidos debido a la creciente masificación del sistema y el ingreso a la educación secundaria de sectores históricamente excluidos, así como la consolidación de un entramado legislativo que reconoce los derechos de los jóvenes, entre ellos, el de su participación política en la construcción de la convivencia escolar.

Por su parte Giroux (1993) sostiene que la ciudadanía debe ser congruente con los postulados de una democracia crítica, pues una ciudadanía desde 
una perspectiva crítica y emancipatoria implica «un proceso de diálogo y compromiso arraigados en una creencia fundamental en la posibilidad de vida pública en el desarrollo de formas de solidaridad» (Giroux 1993:17). Se trata, al decir del autor, de un proceso de producción cultural que sea referencia para la «eliminación de aquellas condiciones ideológicas y materiales que fomentan diversos modos de subyugación, segregación, brutalidad y marginación, frecuentemente expresada por medio de formas sociales que encarnan intereses raciales, clasistas y sexistas» (Giroux 1993:21) y que colaboren a la conformación de relaciones no enajenantes. En tal sentido, el ejercicio de la ciudadanía crítica en la escuela debería poner en cuestión las relaciones de poder y los conflictos que de ellas se desprenden (Hernandez 2013).

Referir al ejercicio de ciudadanía desde una perspectiva emancipatoria que apunte a consolidar la perspectiva de derechos nos remite a pensar la participación desde la politicidad, es decir, lo político en la escuela atendiendo a la dimensión de conflictividad y antagonismo, la dimensión de bien común y de espacio público (Larrondo 2013).

\section{La participación de las y los jóvenes en espacios escolares}

Existen diversidad de formas, situaciones, repertorios en cuanto a la participación de las y los jóvenes en las escuelas secundarias (Nuñez 2010, Larrondo 2013). La modalidad clásica a través de los centros de estudiantes enmarca formas de participación pero no las agotan. Nos preguntamos entonces: ¿Qué procesos de construcción de derechos se configuran en escuelas insertas en contextos desiguales? ¿Cuáles son las prácticas que los y las jóvenes estudiantes realizan en relación a esta perspectiva? ¿Qué sentidos construyen sobre las prácticas institucionalizadas como ampliadoras o potenciadoras de derechos?

Presentaremos aquí resultados parciales de un estudio realizado en escuelas secundarias privadas de una ciudad intermedia de la provincia de Córdoba durante el año 2018. Entendemos que prácticas y sentidos son parte de una misma trama que despliegan los actores intervinientes en la vida escolar, por 
(6) El estudio se desarrolló en tres escuelas privadas de una ciudad intermedia de la provincia de Córdoba. Si bien todas ellas son de gestión privada difieren entre sí pues están insertas en contextos desiguales. Dos de ellas (privadas no confesional) fueron creadas en la década del ' 90 orientadas a recibir a sectores medios y medios altos de la ciudad. Este tipo de escuelas en general proponen proyectos pedagógicos identitarios cimentados en la formación crítica intelectual, la formación humanística y artística, a veces con tintes innovadores.

La población estudiantil que mayoritariamente asiste a estas escuelas son hijos de profesionales, pequeños empresarios, trabajadores formales. Una de ellas situada aproximadamente a $8 \mathrm{~km}$. el centro, se halla anclada en la zona neurálgica del cordón industrial de la ciudad, la otra institución está ubicada en el macro-centro. A ambas asisten jóvenes que provienen de diferentes puntos de la localidad, pues su población busca un tipo de perfil institucional educativo específico. La otra escuela es un Anexo de modalidad rural en espacio urbano y funciona como anexo de una escuela confesional. Está inserta en un barrio popular, ubicada en la periferia urbana al sureste de la ciudad. En general asisten jóvenes del mismo barrio o de las cercanías cuyas condiciones de vida son de vulnerabilidad, muchos de ellos fueron excluidos de otras escuelas. Esta institución creada en 2008 es el resultado eso para conocer los sentidos que los y las jóvenes construyen en relación con los derechos en la escuela, se estudió particularmente las prácticas de los Centros de Estudiantes Secundarios (en adelante CES) y las Asambleas Escolares (en adelante AE). Para ello se utilizó la perspectiva metodológica etnográfica, desde la cual se realizaron entrevistas grupales a estudiantes, observación de espacios de participación y análisis de documentos (actas de reuniones, producciones como consignas y participación en las redes sociales) en las tres escuelas en donde desarrollamos el proyecto de investigación ya mencionado. El trabajo de campo fue realizado durante el ciclo lectivo de 2018. En el caso de los CES se procedió a una entrevista grupal semi-estructurada a los miembros cada uno de estos espacios. Para el caso de las AE (espacio institucionalizado en una de las escuelas ) se consumó un trabajo de campo de tipo etnográfico a través del cual se observaron un total de 9 asambleas realizadas en los tres primeros años de la escuela (turno mañana). Se efectuaron además 6 entrevistas grupales semiestructuradas a estudiantes delegados de todos los cursos de la escuela. La selección de las escuelas sigue un criterio de muestreo teórico según el perfil institucional y las condiciones socio-económicas de su población y el tipo de gestión privada. Todas asumían el enfoque de derechos en sus proyectos institucionales, pero además representan poblaciones escolares diferentes y desiguales entre sí. A una de las instituciones concurren sectores medios altos, a otra concurren sectores medios y trabajadores y a la otra institución asisten jóvenes de sectores populares ${ }^{(6)}$.

\section{Sobre los centros de estudiantes}

¿Cuáles son prácticas que los y las jóvenes llevan a cabo en los $\mathrm{CES}^{(7)}$ ?, ¿Qué finalidades le atribuyen a estos espacios? ¿Para qué participan? ¿Qué significaciones se construyen? Pudimos advertir que los y las jóvenes realizan una multiplicidad de prácticas en estos espacios de participación, a las que hemos agrupado en: prácticas organizativas para sostener estos espacios de participación (elecciones), prácticas cooperativas con las instituciones (arreglos edilicios, cobertura de materiales, etc.) y prácticas de asistencia 
y/o participación social (apoyo escolar, visitas a geriátricos). También desde los centros se realizan prácticas a las que denominamos "de mediación", desde las cuales se interviene para resolver conflictos entre pares, entre estudiantes, docentes y autoridades (situaciones particulares y conflictivas con algún docente, negociaciones con las autoridades).

A partir de las entrevistas y las observaciones hemos advertido cómo las prácticas en los CES se encuentran atravesadas por el impulso y sostén de los adultos. Por ej. en aquellas de tipo organizativas para garantizar la continuidad de los CES, se entablan relaciones de tutela, acompañamiento y promoción por parte de los docentes, coordinadores/ tutores o directivos. En este sentido aparece como muy importante la promoción y sostenimiento que las instituciones dan a la permanencia y hasta la existencia de los CES de cada escuela; este escenario se corresponde también con el propio marco normativo(8) que contempla la presencia de un profesor asesor para potenciar su funcionamiento. Esto parece conjugarse con significaciones por parte de los y las estudiantes: las instituciones "garantizan todo", "gracias a la escuela podemos participar». Se trama a su vez, sedimentos de construcciones hegemónicas arraigadas en muchos de los adultos sobre los y las jóvenes como seres inmaduros, irracionales, vulnerables a partir de las cuales se justifican ciertas prácticas proteccionistas de tutela y acompañamiento. En otras prácticas la incidencia de los adultos es más implícita y se evidencia en cómo los y las estudiantes asumen intereses y discursos de profesores y equipos de gestión institucional. Expresado por los mismos estudiantes como: «la institución nos pide que colaboremos en...», «hay muy buena onda, nosotros colaboramos con todo lo que los profes nos sugieren", "tenemos suerte que en esta escuela podemos participar» (Estudiantes $5^{\circ}$ año CES). Este tipo de prácticas analizadas son consonantes con los hallazgos del estudio de Larrondo (2013). En estas prácticas de actuar mancomunado y de tipo solidarias, lo participativo se esgrime «como único o principal articulador de las acciones colectivas» (Larrondo 2013: 56), aunque quizás carente de potencialidad política. Se evidencia también en todos los CES un fuerte lazo de pertenencia institucional, que imprime un sello en propósitos y horizontes de cada grupo estudiantil. Los sentidos del ejercicio de la ciudadanía a través de este tipo acciones quizás construya de una historia de proyectos socioeducativos barriales desde la militancia social y luego institucionalizada y amparada bajo la órbita privada. Asisten en general hijos de familias con trabajo informal, algunas de ellos ligados a la economía popular.

(7) $\mathrm{La} \mathrm{LEN}\left(\mathrm{N}^{\circ} 26.206\right)$ reconoce la importancia de la participación de los estudiantes: art. I26, inciso $\mathrm{h}$, menciona que las y los alumnas/os tienen derecho a: «Integrar centros, asociaciones y clubes de estudiantes $\mathrm{u}$ otras organizaciones comunitarias para participar en el funcionamiento de las instituciones educativas, con responsabilidades progresivamente mayores, a medida que avancen en los niveles del sistema». A su vez la ley Nacional $\mathrm{N}^{\circ} 26877$ /2013 promueve la creación de los CES reconociendo el valor de la participación en el funcionamiento democrático de las instituciones. De esta manera se debe promover y garantizar la existencia de estos espacios. A su vez el Ministerio de Educación de la provincia de Córdoba a partir de la Resolución $\mathrm{N}^{\circ}$ I24 de 20Io, autoriza la constitución y funcionamiento de un organismo de representación estudiantil en las escuelas de la provincia. En ella además se aprueba el Estatuto Modelo para Centros de Estudiantes. (8) Res I24/Io Anexo. Art. 4I. El Centro de Estudiantes contará con el asesoramiento de un profesor del establecimiento en calidad de asesor, quien lo asistirá tanto en la observancia de 
la normativa vigente y en la correcta determinación de su contabilidad, y colaborará en todos aquellos aspectos que él considere necesario para el mejor cumplimiento de las funciones propias del Centro de Estudiantes. un sujeto participativo, solidario en un clima escolar que se caracteriza por «la buena onda». Estas prácticas pueden ser tributarias de una matriz escolar que invisibiliza la naturaleza política de los antagonismos y conflictos, negando así la capacidad de agencia de los y las jóvenes que los ubica en una situación de apoliticidad y minoridad. (Falconi 2004)

Así pues, hay en las prácticas y en las "cosas dichas», un ejercicio ciudadano que, en la escuela, se presenta como "ensayo», como preparación para la vida adulta, con regulaciones que condensan un modo correcto de participar, donde todo está «dado», «concedido» por los adultos. En consonancia con lo planteado por Hernandez (2013), consideramos que la escuela no puede ser pensada como laboratorio de ejercicio de la ciudadanía, como un entorno protegido o antesala para la vida adulto, sino como un creciente involucramiento en los problemas públicos por parte de los y las jóvenes. Observamos cómo la autonomía, en los procesos participativos, se ve «inducida» por visiones adultocentristas, teñida por las necesidades escolares y quizás por una cosmovisión neoconservadora que se expresa en los límites que las instituciones generan para la participación escolar. Recurrimos aquí a pensar este tipo de prácticas en la escuela a partir de la figura de «ciudadanía instrumental» (Nuñez 2019), figura que, según el autor, cristaliza un modo de hacer política, en este caso realizando tareas colaborativas o compensando algunas de las actividades que le competen a la institución. Además Nuñez y Litichever (2015) señalan cómo las instituciones estructuran límites y posibilidades con las que los y las jóvenes cuentan en el momento de exigir o presentar demandas. Existen también tensiones entre los marcos normativos habilitantes a la participación política escolar (leyes, resoluciones, políticas públicas, espacios de participación juvenil) y la persistencia de resabios de temor a la política partidaria dentro de la escuela. En el actual contexto de reemergencia neoconservadora es posible aventurar que la resolución de estas tensiones se puede orientar en una dirección opuesta a la ampliación y ensanchamiento de las posibilidades que los y las jóvenes tienen para materializar la participación como ejercicio de derechos y construcción de ciudadanía escolar.

También identificamos otro tipo de prácticas que suponen procesos de participación y que emergen de la agenda estudiantil, como las propuestas 
de formación y discusión de temáticas consideradas de actualidad. Son desarrolladas en el espacio institucional aunque con el recaudo de no asumir explícitos posicionamientos políticos partidarios. Para todos los miembros de los centros estos son espacios para debatir y para elaborar proyectos de acuerdo a sus propios intereses pero intentando evitar controversias y confrontaciones entre el colectivo estudiantil y la institución, primando así los intereses comunes en resguardo de una cierta neutralidad (Larrondo 2013). Aunque no de manera generalizada podemos advertir algunos límites que los propios estudiantes ponen a temáticas y posturas que puedan generar controversias con el mundo adulto (docentes, padres) o con los mismos pares. Estos mismos jóvenes, con el propósito de no generar desacuerdos en el espacio escolar, sobrellevan participaciones por fuera de la escuela, en espacios donde sí asumen posiciones políticas (por ej. En la lucha por la despenalización del aborto, Ni una menos, Marcha de la Gorra). Como expresa Reguillo Cruz (2000) son formas de participación desde abajo que escapan a las formas tradicionales de concebir el ejercicio político y a sus escenarios habituales y que expresan un encuentro de intereses entre condición estudiantil y condición juvenil (Reguillo Cruz 2008). En este punto nos preguntamos qué alcance disruptivo tienen este tipo de prácticas que emergen en escenarios no escolares pero mantienen y responden a la lógica hegemónica de concebir la escuela y sus espacios de representación desde la neutralidad. Podríamos decir que esa polisemia con la que se comprende la perspectiva de derechos (Barna 2013) y la participación en la escuela se condensa en estas tensiones entre prácticas atravesadas por regulaciones institucionales y aquellas que parecen expresar mayor autonomía. No obstante, esta delimitación no escapa a las contradicciones propias de todo proceso social en donde se traman dinámicamente los avances y retrocesos del ejercicio de la ciudadanía en la escuela.

En una de las escuelas que asisten jóvenes de clase media reparamos en una práctica que, a nuestro entender, se presenta como disruptiva entre el CES y la institución. Uno de los centros protagonizó tensiones con la dirección escolar en relación con la implementación de las pruebas «Aprender». Los y las estudiantes cuestionaron la implementación de la prueba, cuestión que se articuló con otros CES a través de la UES(9). La negativa a realizar
(9) Unión de Estudiantes Secundarios. Espacio que nuclea a varios centros de estudiantes de las escuelas secundarias de la ciudad fundamentalmente identificadas con el campo popular. 
la prueba se fundamentaba en que la misma era expresión de las reformas neoliberales planificadas para el secundario, situación que enfrentó al CES con las autoridades escolares. Como resultado de esta tensión se dejó a voluntad de cada uno participar o no del operativo de evaluación. Nos preguntamos si este camino resolutivo fue producto de una estrategia disuasiva de la institución o producto de una negociación con las autoridades. La incomodidad "política» que generó este posicionamiento fue advertido por ellos mismos como una de las únicas cuestiones en donde «hubo tironeos y tensiones» con la institución. (Estudiante 6 to año del CES). Subyace a esta práctica la "potencialidad de una conflictividad y de una direccionalidad hacia las autoridades y/o el espacio público» (Larrondo 2013: 53).

La participación así parece estar facilitada y a la vez regulada por el marco institucional, es decir el derecho a la participación es cedido pero, a la vez, tutelado. De esta manera, quizás las fronteras entre autonomía y tutela se encuentran en tensión, las significaciones que se desprenden de cómo los y las jóvenes significan la participación tensiona a su vez la perspectiva de derechos.

El puntapié o motivo de origen de estas prácticas, se encuentran en lo que los y las estudiantes consideran «injusticias». Estas se inscriben en las condiciones materiales de los grupos y en las agendas que van surgiendo al calor de los procesos sociales, políticos, culturales del contexto epocal, acciones que parecen encausarse en una participación más autónoma que desborda las prácticas reguladas por «lo escolar» y que expresaría injusticias visibilizadas y de derechos por conquistar.-

Una de las preguntas que orientó nuestro estudio fue cuáles sentidos se construían sobre perspectiva de derechos en contextos escolares atravesados por la desigualdad. Si bien este punto debe profundizarse, hasta el momento hemos identificado algunas diferencias y contrastes sobre todo entre las prácticas de los CES de la escuela inserta en contexto popular y aquellas prácticas de las escuelas de clases medias y medias altas. Concebimos a las desigualdades desde un enfoque multidimensional que abarca tanto las condiciones materiales y objetivas en la distribución de los recursos y el control de acceso a los mismos como a las asimetrías simbólico-subjetivas de producción de las existencias individuales y colectivas (Bourdieu; 1998). 
Por su parte, G. Therborn (2015) distingue tres dimensiones que conforman las desigualdades; la vital, la existencial y la de recursos. En el último ítem ubica a la educación, pues la entiende como uno de los bienes o recursos que contribuyen a la reproducción o producción simbólica-material importante para la vida social.

Si analizamos los procesos de participación en clave de desigualdad podemos problematizar algunos puntos: las diferentes y desiguales condiciones de vida y capitales en juego (Bourdieu 1998) de los y las jóvenes de sectores populares quizás potencie procesos de sostén y acompañamiento institucional ante trayectorias escolares dificultosas, frecuentemente resultantes de procesos de vulneración de derechos y de exclusión social. Los sentidos construidos en torno al valor de la escuela en este sector como: «un lugar de escucha, de comprensión y de diálogo» atraviesa las significaciones sobre la participación y permite quizás potenciar una autonomía progresiva en el ejercicio de los derechos.

Las condiciones materiales y la vivencia de necesidades básicas insatisfechas tiñen también las prácticas de los CES, el reclamo que realizan los y las jóvenes de sector popular en relación con la necesidad de ampliación del espacio escolar ${ }^{(10)}$ (frente a una infraestructura muy limitada); el desarrollo de tareas como realizar un ropero y producción de algún alimento expresan prácticas que responden a necesidades básicas insatisfechas; mientras que en los otros CES algunas de sus tareas implican embellecer y mejorar el espacio cotidiano escolar.

\section{Sobre las Asambleas Escolares}

En una de las escuelas estudiamos particularmente el espacio de las Asambleas escolares $(\mathrm{AE})^{(11)} \mathrm{A}$ partir de las observaciones caracterizamos estos espacios como de socialización escolar, de elaboración de acuerdos y de diálogo entre los distintos actores institucionales. En los primeros años las temáticas abordadas se vinculan con la circulación de información institucional, el conocimiento de normas y reglas de convivencia, y de trabajo escolar: esperar por la palabra, registrar días y horas de examen, información
(10) Las demandas y propuestas que se identificaron en este CES se vinculan con la ampliación del edificio escolar por la restricción del espacio a las que los y las estudiantes perciben como limitante para la inclusión escolar de otros jóvenes del barrio.

(11) Las asambleas fueron un espacio creado por el colectivo docente para potenciar la circulación de la palabra y el diálogo con los y las jóvenes. Desde la creación de la escuela se realizan con mayor sistematicidad en los

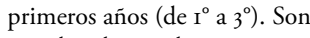
coordinadas por las tutoras -actores claves- encargadas de hacer un seguimiento de tipo socio-afectivo y pedagógico de los estudiantes. También se realiza quincenalmente una asamblea general al inicio de la jornada diaria donde se socializan los temas y problemáticas comunes. La asamblea es un espacio de participación muy propio del campo de la militancia social, según actores institucionales (docentes, tutores y coordinadora) se la recupera como herramienta para potenciar la participación juvenil en la dinámica escolar. 
(12) Quienes coordinan las

AE son los docentes tutores o docentes responsables de las diferentes áreas de conocimientos. sobre materias previas, criterios de evaluación, formas de trabajo estudiantil, expectativas institucionales, etc. Por otra parte se observa, en la dinámica institucional, cómo estos espacios promueven la elaboración de acuerdos ante situaciones de conflictos interpersonales, y ante la necesidad de acordar pautas de trabajo pedagógico y de participación. En los primeros cursos, se identificaron instancias de búsqueda de acuerdos frente a la presencia de distintos conflictos como por ejemplo, problemas de discriminación en algunas clases de educación física, el no cumplimiento de las normas de convivencia dentro del aula, y particularmente en tercer año se abordaron situaciones de tensión en los vínculos interpersonales entre pares. En palabras de algunos estudiantes, "gracias a las asambleas, ya no nos peleamos tanto».

Pudimos percibir cómo prevalece la palabra por parte de quienes coordinan ${ }^{(12)}$ las AE en el primer año. No obstante esta situación va cambiando y se evidencia mayor apropiación y participación de las y los estudiantes en los acuerdos de convivencia en los años más altos.

Nos interesó también indagar las valoraciones de los y las jóvenes sobre este espacio institucional. A partir de las entrevistas reparamos que significan centralmente a las AE como espacios de participación: es el lugar de escucha y de diálogo así como la posibilidad para proponer o reclamar a la escuela asuntos diversos. Expresan sentirse: «fortalecidos», «con derecho a opinar» reconociendo que «nos podemos expresar libremente». Por otra parte, también valoran este espacio por permitir la resolución de conflictos en relación con cuestiones de convivencia escolar entre pares y con adultos de la institución. Se expresa por ejemplo: «ahí arreglamos las cosas cuando nos peleamos» (Estudiantes de $2^{\circ}$ y $3^{\circ}$ año). También reconocen que constituyen espacios de reflexión frente a algunas situaciones cotidianas o coyunturales barriales, locales o de la situación del país. En estos espacios se incorpora la discusión de temas de la agenda social-política actual. Algunos de estos temas son propuestos por el equipo de docentes y otros provienen de los y las estudiantes. "El otro día charlamos el tema de los precios, porque antes comíamos dos comidas ahora no... yo no puedo tomar más coca... yo lo dije a la mañana y hablamos de la inflación y eso» (Estudiante $3^{\circ}$ año). Advertimos la importancia que tiene este espacio para la participación estudiantil y para la construcción de la pertenencia institucional. Los y las 
estudiantes significan a las AE como espacios de democratización escolar, aunque estas asumen en algunos momentos un formato que se acerca a la socialización institucional, pues parecen configurarse en espacios reguladores para la vida cotidiana escolar. Cuando el accionar se centra en promover la convivencia, las experiencias son favorables para recrear un orden escolar desde la apropiación de las normas, como parte del proceso de aprendizaje con participación activa de los y las jóvenes y no una mera inducción o imposición. (Paulin, 2009) Si bien el autor refiere a las distintas modalidades que han asumido los consejos de convivencia podemos trazar similitudes con este tipo de experiencias asamblearias, en relación con la creación de un espacio de diálogo y de ejercicio de derechos.

No obstante, este proceso se vehiculiza a través de una progresiva apropiación de las normas y reglas de convivencia indispensables para favorecer y sostener procesos educativos inclusivos. Según Paulin y Tomassini (2007) se han conformado distintas modalidades institucionales que posibilitan la emergencia de diferentes escenarios de acuerdos de convivencia en donde los actores despliegan prácticas de inducción, resistencia y /o apropiación. Pudimos notar momentos de tensión entre propuestas de estudiantes y el proyecto inclusivo que la escuela sostiene, por ejemplo cuando ante determinados conflictos se reclama a la escuela alguna sanción punitiva para algún compañero. Esta situación tensiona el espacio de la misma asamblea así como el ideario institucional basado en los derechos juveniles. No siempre promover la circulación de la palabra habilita a propuestas democráticas, pues suele arraigar versiones conservadoras del sentido común, sobretodo en esas tramas de significaciones que se despliegan producto de procesos previos de trayectorias escolares: historias de fracasos, abandonos, interrupciones temporarias, sanciones vivenciadas.

Esta modalidad institucional que es un espacio de elaboración de acuerdos para la convivencia puede ser significada como promotora de participación. Quizás sea un dispositivo a partir del cual se favorezca y avance en la concreción del derecho a la educación, y se constituya en un espacio de aprendizaje, ejercicio de convivencia y ciudadanía, pues la apropiación progresiva de las normas básicas institucionales para posibilitar el aprendizaje, la construcción de la pertenencia institucional, la apropiación del espacio, 
la construcción de la valía personal y grupal para proponer, reclamar y ser tomados en cuenta, son algunos de los aspectos que identificamos como fomentados por el espacio asambleario, aun cuando existan tensiones y contradicciones propias de toda dinámica socio-institucional.

\section{A modo de cierre}

La perspectiva de derechos implica un posicionamiento ético y político que debe atravesar las instituciones y los procesos escolares cotidianos. En este marco la construcción de ciudadanía, a través de la participación estudiantil, es una de los nudos centrales para garantizar el ejercicio pleno de los derechos de los y las estudiantes. Como vimos, la participación estudiantil a veces se encauza en espacios institucionalizados, otras veces transita recorridos extraescolares. La construcción de ciudadanía se expresa en una variedad de prácticas, algunas de ellas más cercanas a ampliar la perspectiva de derechos desde un ejercicio autónomo, otras quizás ligadas a las formas tradicionales de ejercicio de ciudadanía escolar.

Esta heterogeneidad conlleva ribetes desiguales pues son expresiones de las desiguales condiciones de vida de los y las jóvenes. Por otra parte, la segmentación de nuestro sistema educativo hace que, también, la construcción de la perspectiva de derechos asuma tintes diferentes según la clase social de la población escolar, pues expresa formas diferentes y desiguales de transitar las experiencias escolares y de construir ciudadanía. Todos estos propósitos disputan sentidos a un contexto de reemergencia neoconservadora que promueve prácticas punitivas a la participación juvenil, criminaliza la protesta social, ataca los movimientos juveniles. No obstante, advertimos también cómo la construcción de la perspectiva de derechos, sus sentidos y significaciones se halla en permanente disputa, asediada por la perspectiva hegemónica pero también batallada por nuevas emergencias contra-hegemónicas que hacen avizorar un horizonte de mayor democratización escolar y social. 


\section{Referencias bibliográficas}

Barna, A. (2013). Los derechos del niño. Un campo en disputa. Boletín de Antropología y Educación volumen 4, (05), 21-25.

Bourdieu, P. (1998). Respuestas. Por una antropología reflexiva. Editorial Grijalbo. Bs. As.Provincia de Córdoba. Res 124/2010. Estatuto Modelo para Centros de Estudiantes. Boletín Oficial AÑO XCVIII - TOMO DXLIII - No 82 CORDOBA, (R.A.) 30/04/10. Recuperado de: 10https://boletinoficial.cba.gov.ar/wp-content/4p96humuzp/2014/09/300410_seccion1.pdf

Falconi, 0. (2004). Las silenciadas batallas juveniles. ¿Quién está marcando el rumbo de la escuela media hoy? Revista de temas sociales, Kairós año 8 №14.

Giroux, H. (1993). La escuela y la lucha por la ciudadanía. Madrid. Siglo XXI Editores.

Hernandez, A. (2013). Ciudadanía, democracia y participación: un estudio de casos en escuelas secundarias de la Ciudad de Córdoba Polifonías. Revista de Educación. Año VII N| 15. 2019. pp 17-53

Larrondo, M. (2013). Escuela Secundaria, Participación Política y Movimiento Estudiantil. Articulaciones conceptuales y actores para el caso de la provincia de Buenos Aires Propuesta Educativa, núm. 39, junio, 2013, pp. 51-58 Facultad Latinoamericana de Ciencias Sociales Buenos Aires, Argentina Recuperado de: http:// www.redalyc.org/articulo.oa?id $=403041710007$

Llobet, V. (2013). La producción de la categoría «niño-sujeto-de-derechos» y el discurso psi en las políticas sociales en Argentina. CLACSO. Pensar la infancia desde América Latina (pp18-209). Recuperado de: http://biblioteca.clacso.edu.ar/clacso/posgrados/20140416023412/PensarLalnfancia.pdf
Magistris, G. (2013). El magnetismo de los derechos: desplazamientos y debates en torno a los derechos de niños, niñas y adolescentes. Serie Documentos de trabajo. Red de posgrados No 40. CLACSO. Recuperado de: http://biblioteca.clacso.edu.ar/clacso/posgrados/20140207020945/Magistris.pdf

Magistris, G. y Morales, S. (2018). Niñez en movimiento: del adultocentrismo a la emancipación. Bs. As. Editorial Chirimbote. El colectivo.

Nuñez, P. (2010): Política y poder en la escuela media. La socialización política juvenil en el espacio escolar. Tesis de doctorado. Universidad Nacional de General Sarmiento- IDES, Buenos Aires.

Núñez, P, y Litichever, L. (2015). Radiografías de la experiencia escolar. Bs.As. Ediciones del Aula Taller.

Núñez, P. (2019). La irrupción de la política en la escuela secundaria: nuevas figuras de ciudadanía. Estudios Sociales. Revista Universitaria Semestral, 56 (1), 155-177.

Fuentes, S, y Núñez, P. (2015). Estudios sobre construcción de ciudadanía en la escuela secundaria argentina: tendencias y categorías en las investigaciones en la última década (2002-2012). Espacios en Blanco, 25, 351-372.

Paulin, H. (2002). Los consejos de convivencia en las escuelas secundarias: sentidos, lecciones y desafíos. Revista Hoy la Universidad, Versión Digital, de la Universidad Nacional de Córdoba, Argentina, setiembre de 2009. recuperado de http://baseries.flacso.org.ar/ uploads/productos/0250_02.pdf 
Paulin, H. y Tomssini, M (2007). Inducción, apropiación y resistencia. Discusiones sobre el concepto de socialización en las prácticas educativas. Revista Conciencia Social. Año VII No 10.UNC.

Paulin, H. y otros (2012). Convivencia e inclusión en las escuelas secundarias: conflictos, innovaciones y rutinas. Anuario de Investigaciones de la Facultad de Psicología, 2012, vol. 1, no 1.

Reguillo Cruz, R. (2000) Emergencias de culturas juveniles. Estrategias del desencanto. Bogotá. Editorial Norma.

Reguillo Cruz, R. (2008). Jóvenes imaginados. La disputa por la representación contra la escencialización. Recuperado de: http://www.revistasbolivianas.org.bo/ pdf/rpc/v13n16/v13n16a02.pdf

Rodríguez, E. (2018).Emputad@s y disputad@s: miradas neoliberales, neoconservadoras y neodesarrollistas sobre I@s jóvenes en América Latina en Vazquez; Ospina. y Domínguez (comp). Juventudes e Infancias en el escenario latinoamericano y caribeño actual. (pp 27-43) Recuperado de http://biblioteca.clacso.edu.ar/ clacso/gt/20180803020740/Juventudes.pdf

Therborn, G. (2015). Los campos de exterminio de la desigualdad. Buenos Aires. Fondo de Cultura Económica. 